Bull. Korean Math. Soc. 52 (2015), No. 2, pp. 661-677

http://dx.doi.org/10.4134/BKMS.2015.52.2.661

\title{
EXISTENCE AND CONCENTRATION RESULTS FOR KIRCHHOFF-TYPE SCHRÖDINGER SYSTEMS WITH STEEP POTENTIAL WELL
}

\author{
Dengfeng Lü
}

ABSTRACT. In this paper, we consider the following Kirchhoff-type Schrödinger system

$$
\left\{\begin{array}{l}
-\left(a_{1}+b_{1} \int_{\mathbb{R}^{3}}|\nabla u|^{2} d x\right) \Delta u+\gamma V(x) u=\frac{2 \alpha}{\alpha+\beta}|u|^{\alpha-2} u|v|^{\beta} \quad \text { in } \mathbb{R}^{3}, \\
-\left(a_{2}+b_{2} \int_{\mathbb{R}^{3}}|\nabla v|^{2} d x\right) \Delta v+\gamma W(x) v=\frac{2 \beta}{\alpha+\beta}|u|^{\alpha}|v|^{\beta-2} v \quad \text { in } \mathbb{R}^{3} \\
u, v \in H^{1}\left(\mathbb{R}^{3}\right),
\end{array}\right.
$$

where $a_{i}$ and $b_{i}$ are positive constants for $i=1,2, \gamma>0$ is a parameter, $V(x)$ and $W(x)$ are nonnegative continuous potential functions. By applying the Nehari manifold method and the concentration-compactness principle, we obtain the existence and concentration of ground state solutions when the parameter $\gamma$ is sufficiently large.

\section{Introduction and main results}

Let us consider the following Kirchhoff-type Schrödinger system in $\mathbb{R}^{3}$ : $(\mathcal{K S})_{\gamma}$

$$
\left\{\begin{array}{l}
-\left(a_{1}+b_{1} \int_{\mathbb{R}^{3}}|\nabla u|^{2} d x\right) \Delta u+\gamma V(x) u=\frac{2 \alpha}{\alpha+\beta}|u|^{\alpha-2} u|v|^{\beta} \quad \text { in } \mathbb{R}^{3} \\
-\left(a_{2}+b_{2} \int_{\mathbb{R}^{3}}|\nabla v|^{2} d x\right) \Delta v+\gamma W(x) v=\frac{2 \beta}{\alpha+\beta}|u|^{\alpha}|v|^{\beta-2} v \quad \text { in } \mathbb{R}^{3} \\
u, v \in H^{1}\left(\mathbb{R}^{3}\right)
\end{array}\right.
$$

where $a_{i}$ and $b_{i}$ are positive constants for $i=1,2, \gamma>0$ is a parameter, $\alpha>2$, $\beta>2$ satisfy $\alpha+\beta<2^{*}=6$, and $V(x), W(x)$ are nonnegative continuous potential functions on $\mathbb{R}^{3}$.

Received May 12, 2014

2010 Mathematics Subject Classification. 35J50, 35J10, 35Q60.

Key words and phrases. Kirchhoff-type Schrödinger system, variational method, concentration, steep potential well. 
In recent years, many papers have extensively considered the scalar Kirchhoff-type equation

$$
\begin{cases}-\left(a+b \int_{\Omega}|\nabla u|^{2} d x\right) \Delta u=f(x, u) & \text { in } \Omega \\ u=0 & \text { on } \partial \Omega\end{cases}
$$

where $a$ and $b$ are positive constants and $\Omega \subset \mathbb{R}^{3}$ is a smooth bounded domain. For example, Ma and Rivera [13] obtained positive solutions of (1.1) by using variational methods. Alves, Corrêa and Ma [1] studied problem (1.1) and obtained positive solutions via the mountain pass theorem. Perera and Zhang [15] obtained a nontrivial solution of (1.1) via Yang index and critical group. Mao and Zhang [14] obtained three solutions by the invariant sets of descent flow. He and Zou [7] showed existence of infinitely many solutions by using the local minimum methods and the fountain theorems. Cheng and $\mathrm{Wu}$ [5] studied the existence of positive solutions for problem (1.1) when the nonlinearity $f$ is asymptotically $t^{3}$-growth at infinity. We also note that problem (1.1) is related to the stationary analogue of the equation

$$
\rho \frac{\partial^{2} u}{\partial t^{2}}-\left(\frac{P_{0}}{h}+\frac{E}{2 L} \int_{0}^{L}\left|\frac{\partial u}{\partial x}\right|^{2} d x\right) \frac{\partial^{2} u}{\partial x^{2}}=0,
$$

where $\rho, P_{0}, h, E, L$ are constants. The equation (1.2) was proposed by Kirchhoff in [9] as an extension of the classical D'Alembert wave equation for free vibrations of elastic strings. Kirchhoff's model considers the changes in length of the string produced by transverse vibrations. Problem (1.2) began to call attention of several researchers after the pioneer work of Lions [10], where a functional analysis approach was proposed. It is pointed in [1] that the problem (1.2) model may describe some physical and biological systems, where $u$ denotes a process which depends on the average of itself.

On the other hand, the following Kirchhoff-type equation

$$
\left\{\begin{array}{l}
-\left(a+b \int_{\mathbb{R}^{3}}|\nabla u|^{2} d x\right) \Delta u+V(x) u=f(x, u) \quad \text { in } \mathbb{R}^{3}, \\
u \in H^{1}\left(\mathbb{R}^{3}\right),
\end{array}\right.
$$

has also been investigated by many authors. In [18], by applying symmetric mountain pass theorem, the author obtained the existence results for nontrivial solutions and a sequence of high energy solutions for problem (1.3). Subsequently, Liu and He [12] proved the existence of infinitely many high energy solutions for (1.3) when $f$ is a subcritical nonlinearity which does't need to satisfy the usual Ambrosetti-Rabinowitz-type growth conditions. More recently, by using variational methods, Sun and $\mathrm{Wu}[16]$ obtained the existence and concentration of ground state solutions for (1.3) when $V(x)$ was replaced by $\lambda V(x)$, where $\lambda$ is a positive parameter. We would also mention the recent papers $[19,20]$ where the existence of high energy solutions for Kirchhoff-type 
Schrödinger systems was established. For more related works, one can also see $[3,4,8,17]$ and the references therein.

Motivated by the works mentioned above, in the present paper we will study a class of Kirchhoff-type Schrödinger systems with steep potential well in $\mathbb{R}^{3}$. Such problems are often referred to as being nonlocal because of the presence of the terms $\left(\int_{\mathbb{R}^{3}}|\nabla u|^{2} d x\right) \Delta u$ and $\left(\int_{\mathbb{R}^{3}}|\nabla v|^{2} d x\right) \Delta v$ which imply that problem $(\mathcal{K S})_{\gamma}$ is no longer pointwise identity. This phenomenon provokes some mathematical difficulties, which motivate the study of such a class of particularly interesting problems. The existence and concentration of ground state solutions of $(\mathcal{K S})_{\gamma}$ are obtained by applying the Nehari manifold method and concentration-compactness principle.

Before stating our main results, we need to introduce some assumptions and notations:

$\left(\mathcal{H}_{1}\right) V(x), W(x) \in \mathcal{C}\left(\mathbb{R}^{3},[0,+\infty)\right)$ and $\Omega:=\operatorname{int}\left(V^{-1}(0)\right)=\operatorname{int}\left(W^{-1}(0)\right)$ is nonempty with smooth boundary and $\bar{\Omega}=V^{-1}(0)=W^{-1}(0)$;

$\left(\mathcal{H}_{2}\right)$ there exist $M_{1}, M_{2}>0$ such that $\mathcal{L}\left(\left\{x \in \mathbb{R}^{3} \mid V(x) \leq M_{1}\right\}\right)<\infty, \mathcal{L}(\{x \in$ $\left.\left.\mathbb{R}^{3} \mid W(x) \leq M_{2}\right\}\right)<\infty$, where $\mathcal{L}$ denotes the Lebesgue measure in $\mathbb{R}^{3}$.

The conditions $\left(\mathcal{H}_{1}\right)$ and $\left(\mathcal{H}_{2}\right)$ imply that $\gamma V(x)$ and $\gamma W(x)$ represent potential well whose depth is controlled by $\gamma . \gamma V(x)$ and $\gamma W(x)$ are called steep potential well if $\gamma$ is sufficiently large, and one expects to find solutions which localize near its bottom $\Omega$. The hypothesis $\left(\mathcal{H}_{2}\right)$ was first introduced by Bartsch and Wang [2] in the study of a nonlinear Schrödinger equation. Let $E_{V}:=\left\{u \in H^{1}\left(\mathbb{R}^{3}\right): \int_{\mathbb{R}^{3}} V(x) u^{2} d x<+\infty\right\}$ and $E_{W}:=\left\{v \in H^{1}\left(\mathbb{R}^{3}\right)\right.$ : $\left.\int_{\mathbb{R}^{3}} W(x) v^{2} d x<+\infty\right\}$ with the norms $\|u\|_{\gamma, V}^{2}=\int_{\mathbb{R}^{3}}\left(a_{1}|\nabla u|^{2}+\gamma V(x) u^{2}\right) d x$ and $\|v\|_{\gamma, W}^{2}=\int_{\mathbb{R}^{3}}\left(a_{2}|\nabla v|^{2}+\gamma W(x) v^{2}\right) d x$ respectively. For any given $\gamma>0$, we consider the Hilbert space $E:=E_{V} \times E_{W}$ endowed with the norm

$$
\|(u, v)\|_{\gamma}=\left(\|u\|_{\gamma, V}^{2}+\|v\|_{\gamma, W}^{2}\right)^{\frac{1}{2}}
$$

The energy functional associated with $(\mathcal{K S})_{\gamma}$ is defined on $E$ by

$$
\mathcal{E}_{\gamma}(u, v)=\frac{1}{2}\|(u, v)\|_{\gamma}^{2}+\frac{1}{4}\left(b_{1} \Upsilon^{2}(u)+b_{2} \Upsilon^{2}(v)\right)-\frac{2}{\alpha+\beta} \int_{\mathbb{R}^{3}}|u|^{\alpha}|v|^{\beta} d x
$$

where $\Upsilon(w)=\int_{\mathbb{R}^{3}}|\nabla w|^{2} d x$. In view of the assumptions $\left(\mathcal{H}_{1}\right)$ and $\left(\mathcal{H}_{2}\right)$, the energy functional $\mathcal{E}_{\gamma}(u, v)$ is well defined and belongs to $\mathcal{C}^{1}(E, \mathbb{R})$, and that

$$
\begin{aligned}
\left\langle\mathcal{E}_{\gamma}^{\prime}(u, v),(\varphi, \psi)\right\rangle= & \left(a_{1}+b_{1} \int_{\mathbb{R}^{3}}|\nabla u|^{2} d x\right) \int_{\mathbb{R}^{3}} \nabla u \nabla \varphi d x \\
& +\left(a_{2}+b_{2} \int_{\mathbb{R}^{3}}|\nabla v|^{2} d x\right) \int_{\mathbb{R}^{3}} \nabla v \nabla \psi d x \\
& +\gamma \int_{\mathbb{R}^{3}}(V(x) u \varphi+W(x) v \psi) d x \\
& -\frac{2}{\alpha+\beta} \int_{\mathbb{R}^{3}}\left(\alpha|u|^{\alpha-2}|v|^{\beta} u \varphi+\beta|u|^{\alpha}|v|^{\beta-2} v \psi\right) d x .
\end{aligned}
$$


Hence, if $(u, v) \in E$ is a critical point of $\mathcal{E}_{\gamma}(u, v)$, then $(u, v)$ is a solution of problem $(\mathcal{K S})_{\gamma}$.

We define the minimax $c_{\gamma}$ as

$$
c_{\gamma}=\inf _{(u, v) \in \mathcal{N}_{\gamma}} \mathcal{E}_{\gamma}(u, v)
$$

where

$$
\mathcal{N}_{\gamma}=\left\{(u, v) \in E \backslash\{(0,0)\}:\left\langle\mathcal{E}_{\gamma}^{\prime}(u, v),(u, v)\right\rangle=0\right\},
$$

$\langle\cdot, \cdot\rangle$ is the duality product between $E$ and its dual space $E^{-1}$. Note that $\mathcal{N}_{\gamma}$ contains every nonzero solution of problem $(\mathcal{K S})_{\gamma}$.

A ground state solution for $(\mathcal{K S})_{\gamma}$ is a critical point $\left(u_{0}, v_{0}\right)$ of $\mathcal{E}_{\gamma}(u, v)$ in $E$ which solves the following minimization problem

$$
\inf _{(u, v) \in \mathcal{N}_{\gamma}} \mathcal{E}_{\gamma}(u, v)=\mathcal{E}_{\gamma}\left(u_{0}, v_{0}\right) .
$$

The main results we get are the following:

Theorem 1.1. Suppose that $\left(\mathcal{H}_{1}\right)$ and $\left(\mathcal{H}_{2}\right)$ hold, then there is $\gamma^{*}>0$ such that, for all $\gamma \geq \gamma^{*}$, the system $(\mathcal{K S})_{\gamma}$ has at least a ground state solution $\left(u_{\gamma}, v_{\gamma}\right)$ in $H^{1}\left(\mathbb{R}^{3}\right) \times H^{1}\left(\mathbb{R}^{3}\right)$.

Theorem 1.2. For each $\gamma>0$ large, let $\left(u_{\gamma}, v_{\gamma}\right)$ be the solutions obtained in Theorem 1.1. Then $\left(u_{\gamma}, v_{\gamma}\right) \rightarrow(u, v)$ as $\gamma \rightarrow \infty$, where $(u, v)$ is a nontrivial solution of

$$
\begin{cases}-\left(a_{1}+b_{1} \int_{\Omega}|\nabla u|^{2} d x\right) \Delta u=\frac{2 \alpha}{\alpha+\beta}|u|^{\alpha-2} u|v|^{\beta} & \text { in } \Omega, \\ -\left(a_{2}+b_{2} \int_{\Omega}|\nabla v|^{2} d x\right) \Delta v=\frac{2 \beta}{\alpha+\beta}|u|^{\alpha}|v|^{\beta-2} v & \text { in } \Omega, \\ u(x)=0, \quad v(x)=0 & \text { on } \partial \Omega .\end{cases}
$$

As far as we know, problem $(\mathcal{K S})_{\gamma}$ has not been considered before. In order to prove the main results, we have to overcome some difficulties in using variational methods. The main difficulties lie in the appearance of the nonlocal term and the lack of compactness due to the unboundedness of the domain $\mathbb{R}^{3}$. Since we neither assume that the potentials are radially symmetric nor impose any other hypotheses on the behavior of the potentials for $|x| \rightarrow \infty$, we can not use the usual way to recover compactness. To recover the compactness, we adopt the idea used in [2] and establish the compactness conditions dependent of parameter. Let us point out that the adaptation of the idea to our problem is not trivial at all because of the presence of the nonlocal terms $\left(\int_{\mathbb{R}^{3}}|\nabla u|^{2} d x\right) \Delta u$, $\left(\int_{\mathbb{R}^{3}}|\nabla v|^{2} d x\right) \Delta v$ and the coupled terms $|u|^{\alpha-2} u|v|^{\beta},|u|^{\alpha}|v|^{\beta-2} v$.

This paper is organized as follows. In Section 2, we will prove some important lemmas that will be used for the proofs of the main results. Section 3 is devoted to the proofs of Theorems 1.1 and 1.2. 


\section{Preliminaries}

Notation. In this paper we will use the following notations:

- $C, C_{1}, C_{2}, \ldots$ denote positive(possibly different) constants.

- $\rightarrow$ (respectively $\rightarrow$ ) denotes strong (respectively weak) convergence.

- $o_{n}(1)$ denotes $o_{n}(1) \rightarrow 0$ as $n \rightarrow \infty$.

- $L^{s}(\Omega)(1 \leq s<+\infty)$ denote Lebesgue spaces, the norm $L^{s}$ is denoted by $|\cdot|_{s}$ for $1 \leq s<+\infty$.

- For given set $K \subset \mathbb{R}^{3}$, we set $K^{c}=\mathbb{R}^{3} \backslash K$.

- $B_{r}$ denotes a ball centered at the origin with radius $r>0$.

- The dual space of a Banach space $E$ will be denoted by $E^{-1}$.

- $\langle\cdot, \cdot\rangle$ denote the duality pairing between $E^{-1}$ and $E$.

The functional $I \in C^{1}(E, \mathbb{R})$ is said to satisfy the $(P S)_{c}$ condition if any sequence $\left\{z_{n}\right\} \subset E$ such that as $n \rightarrow \infty, I\left(z_{n}\right) \rightarrow c, I^{\prime}\left(z_{n}\right) \rightarrow 0$ strongly in $E^{-1}$ contains a subsequence converging in $E$ to a critical point of $I$. In this paper, we will take $I=\mathcal{E}_{\gamma}(u, v)$ and $E=E_{V} \times E_{W}$.

Lemma 2.1. Under the conditions $\left(\mathcal{H}_{1}\right)$ and $\left(\mathcal{H}_{2}\right)$, the following hold:

(i) Let $(u, v) \in \mathcal{N}_{\gamma}$, then there exists $\sigma>0$ which is independent of $\gamma$ such that $\|(u, v)\|_{\gamma} \geq \sigma$.

(ii) For each $(u, v) \in E \backslash\{(0,0)\}$, there is a unique $t_{(u, v)}>0$ such that $t_{(u, v)}(u, v) \in \mathcal{N}_{\gamma}$. Moreover, $\mathcal{E}_{\gamma}\left(t_{(u, v)}(u, v)\right)=\max _{t \geq 0} \mathcal{E}_{\gamma}(t(u, v))$.

Proof. (i) First, by Young inequality, we get

$$
|u|^{\alpha}|v|^{\beta} \leq \frac{\alpha}{\alpha+\beta}|u|^{\alpha+\beta}+\frac{\beta}{\alpha+\beta}|v|^{\alpha+\beta},
$$

then by the continuity of the Sobolev embedding $E_{V} \hookrightarrow L^{s}\left(\mathbb{R}^{3}\right)$ and $E_{W} \hookrightarrow$ $L^{s}\left(\mathbb{R}^{3}\right)$ for $2 \leq s \leq 6$, we obtain

$$
\begin{aligned}
\int_{\mathbb{R}^{3}}|u|^{\alpha}|v|^{\beta} d x & \leq \frac{\alpha}{\alpha+\beta} \int_{\mathbb{R}^{3}}|u|^{\alpha+\beta} d x+\frac{\beta}{\alpha+\beta} \int_{\mathbb{R}^{3}}|v|^{\alpha+\beta} d x \\
& \leq C_{1}\|u\|_{V}^{\alpha+\beta}+C_{2}\|v\|_{W}^{\alpha+\beta} \leq C\|(u, v)\|_{\gamma}^{\alpha+\beta}
\end{aligned}
$$

where $C>0$ is independent of $\gamma$. So, by $(2.1)$, for any $(u, v) \in \mathcal{N}_{\gamma}$, we have

$$
0=\left\langle\mathcal{E}_{\gamma}^{\prime}(u, v),(u, v)\right\rangle=\|(u, v)\|_{\gamma}^{2}+b_{1} \Upsilon^{2}(u)+b_{2} \Upsilon^{2}(v)-2 \int_{\mathbb{R}^{3}}|u|^{\alpha}|v|^{\beta} d x
$$

Note that $\alpha+\beta>4$, thus there exists $\sigma>0$ such that $\|(u, v)\|_{\gamma} \geq \sigma$.

(ii) Let $(u, v) \in E \backslash\{(0,0)\}$ be fixed. For $t>0$, we consider the fibering maps $\phi: t \rightarrow \mathcal{E}_{\gamma}(t(u, v))$ defined by

$$
\phi(t):=\mathcal{E}_{\gamma}(t(u, v))=\frac{t^{2}}{2}\|(u, v)\|_{\gamma}^{2}+\frac{t^{4}}{4}\left(b_{1} \Upsilon^{2}(u)+b_{2} \Upsilon^{2}(v)\right)-\frac{2 t^{\alpha+\beta}}{\alpha+\beta} \int_{\mathbb{R}^{3}}|u|^{\alpha}|v|^{\beta} d x
$$


We observe that $\phi^{\prime}(t)=\left\langle\mathcal{E}_{\gamma}^{\prime}(t(u, v)),(u, v)\right\rangle=0$ if and only if $t(u, v) \in \mathcal{N}_{\gamma}$. First we claim that $\phi(t)>0$ for $t>0$ small. Indeed, by (2.1), we have that

$$
\begin{aligned}
\phi(t) & \geq \frac{t^{2}}{2}\|(u, v)\|_{\gamma}^{2}-\frac{2 t^{\alpha+\beta}}{\alpha+\beta} \int_{\mathbb{R}^{3}}|u|^{\alpha}|v|^{\beta} d x \\
& \geq \frac{t^{2}}{2}\|(u, v)\|_{\gamma}^{2}-C_{3} t^{\alpha+\beta}\|(u, v)\|_{\gamma}^{\alpha+\beta},
\end{aligned}
$$

since $\alpha+\beta>4$, so $\phi(t)>0$ whenever $t>0$ is small enough. It is easy to see that $\phi(t) \rightarrow-\infty$ as $t \rightarrow+\infty$. Hence there exists $t_{(u, v)}>0$ such that $\phi^{\prime}\left(t_{(u, v)}\right)=0$, that is $t_{(u, v)}(u, v) \in \mathcal{N}_{\gamma}$. Moreover, $\mathcal{E}_{\gamma}\left(t_{(u, v)}(u, v)\right)=\max _{t \geq 0} \mathcal{E}_{\gamma}(t(u, v))$.

In addition, the condition $\phi^{\prime}(t)=0$ is equivalent to

$$
b_{1} \Upsilon^{2}(u)+b_{2} \Upsilon^{2}(v)=-\frac{1}{t^{2}}\|(u, v)\|_{\gamma}^{2}+2 t^{\alpha+\beta-4} \int_{\mathbb{R}^{3}}|u|^{\alpha}|v|^{\beta} d x .
$$

The right side of (2.3) is strictly increasing for $t>0$ recalling that $\alpha+\beta>4$, so there exists a unique $t_{(u, v)}>0$ such that $\phi^{\prime}\left(t_{(u, v)}\right)=0$, and the second conclusion follows.

Lemma 2.2. Let $\left(\mathcal{H}_{1}\right)-\left(\mathcal{H}_{2}\right)$ hold and $\left\{\left(u_{n}, v_{n}\right)\right\}$ is a $(P S)_{c}$ sequence for $\mathcal{E}_{\gamma}(u, v)$. Then we have

(i) $\left\{\left(u_{n}, v_{n}\right)\right\}$ is bounded in $E$;

(ii) if $c \neq 0$, then $c \geq c_{0}$, for some $c_{0}>0$ is independent of $\gamma$.

Proof. Let $\left\{\left(u_{n}, v_{n}\right)\right\}$ be a $(P S)_{c}$ sequence for $\mathcal{E}_{\gamma}(u, v)$, that is,

$$
\mathcal{E}_{\gamma}\left(u_{n}, v_{n}\right)=c+o_{n}(1) \text { and } \mathcal{E}_{\gamma}^{\prime}\left(u_{n}, v_{n}\right)=o_{n}(1) .
$$

Then we have,

$c+o_{n}(1)-\frac{1}{4} o_{n}\left(\left\|\left(u_{n}, v_{n}\right)\right\|_{\gamma}\right)=\mathcal{E}_{\gamma}\left(u_{n}, v_{n}\right)-\frac{1}{4}\left\langle\mathcal{E}_{\gamma}^{\prime}\left(u_{n}, v_{n}\right),\left(u_{n}, v_{n}\right)\right\rangle$

$$
\begin{aligned}
& =\frac{1}{2}\left\|\left(u_{n}, v_{n}\right)\right\|_{\gamma}^{2}+\left(\frac{1}{2}-\frac{2}{\alpha+\beta}\right) \int_{\mathbb{R}^{3}}\left|u_{n}\right|^{\alpha}\left|v_{n}\right|^{\beta} d x \\
& \geq \frac{1}{2}\left\|\left(u_{n}, v_{n}\right)\right\|_{\gamma}^{2},
\end{aligned}
$$

which implies that $\left\{\left(u_{n}, v_{n}\right)\right\}$ is bounded in $E$.

On the other hand, we have

$$
\begin{aligned}
o_{n}\left(\left\|\left(u_{n}, v_{n}\right)\right\|_{\gamma}\right) & =\left\langle\mathcal{E}_{\gamma}^{\prime}\left(u_{n}, v_{n}\right),\left(u_{n}, v_{n}\right)\right\rangle \\
& =\left\|\left(u_{n}, v_{n}\right)\right\|_{\gamma}^{2}+b_{1} \Upsilon^{2}\left(u_{n}\right)+b_{2} \Upsilon^{2}\left(v_{n}\right)-2 \int_{\mathbb{R}^{3}}\left|u_{n}\right|^{\alpha}\left|v_{n}\right|^{\beta} d x \\
& \geq\left\|\left(u_{n}, v_{n}\right)\right\|_{\gamma}^{2}-2 C\left\|\left(u_{n}, v_{n}\right)\right\|_{\gamma}^{\alpha+\beta},
\end{aligned}
$$


since $\alpha+\beta>4$, there exists $0<\sigma_{1}<1$ such that

$$
\left\langle\mathcal{E}_{\gamma}^{\prime}\left(u_{n}, v_{n}\right),\left(u_{n}, v_{n}\right)\right\rangle \geq \frac{1}{4}\left\|\left(u_{n}, v_{n}\right)\right\|_{\gamma}^{2} \text { for }\left\|\left(u_{n}, v_{n}\right)\right\|_{\gamma}<\sigma_{1} .
$$

Now, if $c<\frac{\sigma_{1}^{2}}{2}$ and $\left\{\left(u_{n}, v_{n}\right)\right\}$ is a $(P S)_{c}$-sequence of $\mathcal{E}_{\gamma}$, then by $(2.4)$

$$
\lim _{n \rightarrow \infty}\left\|\left(u_{n}, v_{n}\right)\right\|_{\gamma}^{2} \leq 2 c<\sigma_{1}^{2} \text {. }
$$

Hence, $\left\|\left(u_{n}, v_{n}\right)\right\|_{\gamma}<\sigma_{1}$ for $n$ large, then by (2.5)

$$
\frac{1}{4}\left\|\left(u_{n}, v_{n}\right)\right\|_{\gamma}^{2} \leq\left\langle\mathcal{E}_{\gamma}^{\prime}\left(u_{n}, v_{n}\right),\left(u_{n}, v_{n}\right)\right\rangle=o_{n}(1)\left\|\left(u_{n}, v_{n}\right)\right\|_{\gamma},
$$

which implies $\left\|\left(u_{n}, v_{n}\right)\right\|_{\gamma} \rightarrow 0$ as $n \rightarrow \infty$ and $c=0$. It follows that (ii) holds for $c_{0}=\frac{\sigma_{1}^{2}}{2}$.

Lemma 2.3. Let $C^{*}$ be fixed. Given $\varepsilon>0$ there exist $\Gamma_{\varepsilon}=\Gamma\left(\varepsilon, C^{*}\right)>0$ and $\rho_{\varepsilon}=\rho\left(\varepsilon, C^{*}\right)>0$ such that, if $\left\{\left(u_{n}, v_{n}\right)\right\}$ is a $(P S)_{c}$-sequence of $\mathcal{E}_{\gamma}(u, v)$ with $c \leq C^{*}, \gamma \geq \Gamma_{\varepsilon}$, then

$$
\limsup _{n \rightarrow \infty} \int_{B_{\rho_{\varepsilon}}^{c}}\left|u_{n}\right|^{\alpha}\left|v_{n}\right|^{\beta} d x \leq \varepsilon .
$$

Proof. For $\rho>0$, we set

$A(\rho):=\left\{x \in \mathbb{R}^{3}:|x| \geq \rho, V(x) \geq M_{1}\right\}, B(\rho):=\left\{x \in \mathbb{R}^{3}:|x| \geq \rho, V(x)<M_{1}\right\}$, then

$$
\begin{aligned}
\int_{A(\rho)}\left|u_{n}\right|^{2} d x & \leq \frac{1}{\gamma M_{1}} \int_{\mathbb{R}^{3}} \gamma V(x) u_{n}^{2} d x \\
& \leq \frac{1}{\gamma M_{1}} \int_{\mathbb{R}^{3}}\left(a_{1}\left|\nabla u_{n}\right|^{2}+\gamma V(x) u_{n}^{2}\right) d x \\
& \leq \frac{1}{\gamma M_{1}}\left(2 c+o_{n}\left(\left\|\left(u_{n}, v_{n}\right)\right\|_{\gamma}\right)\right) \\
& \leq \frac{1}{\gamma M_{1}}\left(2 C^{*}+o_{n}\left(\left\|\left(u_{n}, v_{n}\right)\right\|_{\gamma}\right)\right) \rightarrow 0 \quad \text { as } \gamma \rightarrow \infty
\end{aligned}
$$

Using the Hölder inequality and (2.4), for $1<q<3$ we obtain

$$
\begin{aligned}
\int_{B(\rho)}\left|u_{n}\right|^{2} d x & \leq\left(\int_{\mathbb{R}^{3}}\left|u_{n}\right|^{2 q} d x\right)^{\frac{1}{q}} \cdot \mathcal{L}(B(\rho))^{\frac{q-1}{q}} \\
& \leq C_{4}\left\|u_{n}\right\|_{H^{1}\left(\mathbb{R}^{3}\right)}^{2} \cdot \mathcal{L}(B(\rho))^{\frac{q-1}{q}} \\
& \leq C_{4} \cdot 2 C^{*} \cdot \mathcal{L}(B(\rho))^{\frac{q-1}{q}} \rightarrow 0 \quad \text { as } \rho \rightarrow \infty
\end{aligned}
$$


where $C_{4}=C_{4}(q)$ is a positive constant. Setting $\theta=\frac{3(\alpha+\beta-2)}{2(\alpha+\beta)}$, by using the Gagliardo-Nirenberg inequality, (2.7) and (2.8), we obtain that

$$
\begin{aligned}
\int_{B_{\rho}^{c}}\left|u_{n}\right|^{\alpha+\beta} d x & \leq C\left(\int_{B_{\rho}^{c}}\left|\nabla u_{n}\right|^{2} d x\right)^{\frac{(\alpha+\beta) \theta}{2}} \cdot\left(\int_{B_{\rho}^{c}}\left|u_{n}\right|^{2} d x\right)^{\frac{(\alpha+\beta)(1-\theta)}{2}} \\
& \leq C_{5}\left\|\left(u_{n}, v_{n}\right)\right\|_{\gamma}^{(\alpha+\beta) \theta} \cdot\left(\int_{A(\rho)}\left|u_{n}\right|^{2} d x+\int_{B(\rho)}\left|u_{n}\right|^{2} d x\right)^{\frac{(\alpha+\beta)(1-\theta)}{2}} \\
& \leq C_{6}\left(\int_{A(\rho)}\left|u_{n}\right|^{2} d x+\int_{B(\rho)}\left|u_{n}\right|^{2} d x\right)^{\frac{(\alpha+\beta)(1-\theta)}{2}} \rightarrow 0 \text { as } \gamma, \rho \rightarrow \infty .
\end{aligned}
$$

Similarly,

$$
\int_{B_{\rho}^{c}}\left|v_{n}\right|^{\alpha+\beta} d x \leq \varepsilon \quad \text { for } \gamma, \rho \text { large. }
$$

At last, using the Hölder inequality, (2.9) and (2.10) we have that

$$
\begin{aligned}
& \limsup _{n \rightarrow \infty} \int_{B_{\rho_{\varepsilon}}^{c}}\left|u_{n}\right|^{\alpha}\left|v_{n}\right|^{\beta} d x \\
\leq & \limsup _{n \rightarrow \infty}\left(\int_{B_{\rho_{\varepsilon}}^{c}}\left|u_{n}\right|^{\alpha+\beta} d x\right)^{\frac{\alpha}{\alpha+\beta}}\left(\int_{B_{\rho_{\varepsilon}}^{c}}\left|v_{n}\right|^{\alpha+\beta} d x\right)^{\frac{\beta}{\alpha+\beta}} \leq \varepsilon .
\end{aligned}
$$

This concludes the proof of Lemma 2.3.

The following Brézis-Lieb type lemma is proved in [6].

Lemma 2.4. Let $\alpha+\beta<2^{*}$ and $\left\{\left(u_{n}, v_{n}\right)\right\} \subset E$ is a sequence such that $\left(u_{n}, v_{n}\right) \rightarrow(u, v)$ weakly in $E$. Then we have

$$
\int_{\mathbb{R}^{3}}\left|u_{n}\right|^{\alpha}\left|v_{n}\right|^{\beta} d x-\int_{\mathbb{R}^{3}}\left|u_{n}-u\right|^{\alpha}\left|v_{n}-v\right|^{\beta} d x=\int_{\mathbb{R}^{3}}|u|^{\alpha}|v|^{\beta} d x+o_{n}(1) .
$$

Lemma 2.5. Let $\gamma>0$ be fixed and $\left\{\left(u_{n}, v_{n}\right)\right\}$ is a $(P S)_{c}$-sequence of $\mathcal{E}_{\gamma}$. Then up to a subsequence $\left(u_{n}, v_{n}\right) \rightarrow(u, v)$ in $E$ with $(u, v)$ being a weak solution of $(\mathcal{K S})_{\gamma}$. Moreover, $\left\{\left(u_{n}-u, v_{n}-v\right)\right\}$ is a $(P S)_{d}$-sequence for $\mathcal{E}_{\gamma}$ with $d=c-\mathcal{E}_{\gamma}(u, v)$.

Proof. Since $\left\{\left(u_{n}, v_{n}\right)\right\}$ is bounded in $E$ (see Lemma 2.2(i)), there is a subsequence of $\left\{\left(u_{n}, v_{n}\right)\right\}$ such that $\left(u_{n}, v_{n}\right) \rightarrow(u, v)$ in $E$ as $n \rightarrow \infty$. In order to see that $(u, v)$ is a critical point of $\mathcal{E}_{\gamma}$ we recall that

$$
\begin{cases}\left(u_{n}, v_{n}\right) \rightarrow(u, v), & \text { in } E, \\ \left(u_{n}, v_{n}\right) \rightarrow(u, v), & \text { a.e. in } \mathbb{R}^{3}, \\ \left(u_{n}, v_{n}\right) \rightarrow(u, v), & \text { in } L_{l o c}^{s_{1}}\left(\mathbb{R}^{3}\right) \times L_{l o c}^{s_{2}}\left(\mathbb{R}^{3}\right), 2 \leq s_{1}, s_{2}<6 .\end{cases}
$$

Moreover, there exist $A, B \in \mathbb{R}$, such that

$$
\int_{\mathbb{R}^{3}}\left|\nabla u_{n}\right|^{2} d x \rightarrow A, \quad \int_{\mathbb{R}^{3}}\left|\nabla v_{n}\right|^{2} d x \rightarrow B,
$$


then by the Fatou's lemma we get that

$$
\int_{\mathbb{R}^{3}}|\nabla u|^{2} d x \leq A, \quad \int_{\mathbb{R}^{3}}|\nabla v|^{2} d x \leq B .
$$

We claim that $\int_{\mathbb{R}^{3}}|\nabla u|^{2} d x=A$, and $\int_{\mathbb{R}^{3}}|\nabla v|^{2} d x=B$. Arguing by contradiction, we assume that $\int_{\mathbb{R}^{3}}|\nabla u|^{2} d x<A$ or $\int_{\mathbb{R}^{3}}|\nabla v|^{2} d x<B$. By $\mathcal{E}_{\gamma}^{\prime}\left(u_{n}, v_{n}\right) \rightarrow 0$ and $\left(u_{n}, v_{n}\right) \rightarrow(u, v)$ in $E$, for any $(\varphi, \psi) \in E$, we have

$$
\begin{aligned}
& \int_{\mathbb{R}^{3}}\left(a_{1} \nabla u \nabla \varphi+a_{2} \nabla v \nabla \psi\right) d x+\gamma \int_{\mathbb{R}^{3}} V(x) u \varphi+W(x) v \psi d x+A b_{1} \int_{\mathbb{R}^{3}} \nabla u \nabla \varphi d x \\
& +B b_{2} \int_{\mathbb{R}^{3}} \nabla v \nabla \psi d x-\frac{2}{\alpha+\beta} \int_{\mathbb{R}^{3}}\left(\alpha|u|^{\alpha-2} u|v|^{\beta} \varphi+\beta|u|^{\alpha}|v|^{\beta-2} v \psi\right) d x=0 .
\end{aligned}
$$

Then $\left\langle\mathcal{E}_{\gamma}^{\prime}(u, v),(u, v)\right\rangle<0$. On the other hand, by Lemma 2.1(ii) it is easy to get that $\left\langle\mathcal{E}_{\gamma}^{\prime}(t(u, v)), t(u, v)\right\rangle>0$ for $t>0$ is small enough. Hence there exists $t_{0} \in(0,1)$ satisfying $\left\langle\mathcal{E}_{\gamma}^{\prime}\left(t_{0}(u, v)\right), t_{0}(u, v)\right\rangle=0$. Moreover, $\mathcal{E}_{\gamma}\left(t_{0}(u, v)\right)=$ $\max _{0 \leq t \leq 1} \mathcal{E}_{\gamma}(t(u, v))$, so

$$
\begin{aligned}
c_{\gamma} & \leq \mathcal{E}_{\gamma}\left(t_{0}(u, v)\right)=\mathcal{E}_{\gamma}\left(t_{0}(u, v)\right)-\frac{1}{4}\left\langle\mathcal{E}_{\gamma}^{\prime}\left(t_{0}(u, v)\right), t_{0}(u, v)\right\rangle \\
& =\frac{t_{0}^{2}}{4}\|(u, v)\|_{\gamma}^{2}+\left(\frac{1}{2}-\frac{2}{\alpha+\beta}\right) t_{0}^{\alpha+\beta} \int_{\mathbb{R}^{3}}|u|^{\alpha}|v|^{\beta} d x \\
& <\frac{1}{4}\|(u, v)\|_{\gamma}^{2}+\left(\frac{1}{2}-\frac{2}{\alpha+\beta}\right) \int_{\mathbb{R}^{3}}|u|^{\alpha}|v|^{\beta} d x \\
& \leq \liminf _{n \rightarrow \infty}\left(\frac{1}{4}\left\|\left(u_{n}, v_{n}\right)\right\|_{\gamma}^{2}+\left(\frac{1}{2}-\frac{2}{\alpha+\beta}\right) \int_{\mathbb{R}^{3}}\left|u_{n}\right|^{\alpha}\left|v_{n}\right|^{\beta} d x\right) \\
& =\liminf _{n \rightarrow \infty}\left(\mathcal{E}_{\gamma}\left(u_{n}, v_{n}\right)-\frac{1}{4}\left\langle\mathcal{E}_{\gamma}^{\prime}\left(u_{n}, v_{n}\right),\left(u_{n}, v_{n}\right)\right\rangle\right)=c_{\gamma},
\end{aligned}
$$

which is a contradiction. Then

$\int_{\mathbb{R}^{3}}|\nabla u|^{2} d x=A=\lim _{n \rightarrow \infty} \int_{\mathbb{R}^{3}}\left|\nabla u_{n}\right|^{2} d x, \int_{\mathbb{R}^{3}}|\nabla v|^{2} d x=B=\lim _{n \rightarrow \infty} \int_{\mathbb{R}^{3}}\left|\nabla v_{n}\right|^{2} d x$.

Thus for any $(\varphi, \psi) \in E$, we have

$$
\left\langle\mathcal{E}_{\gamma}^{\prime}(u, v),(\varphi, \psi)\right\rangle=\lim _{n \rightarrow \infty}\left\langle\mathcal{E}_{\gamma}^{\prime}\left(u_{n}, v_{n}\right),(\varphi, \psi)\right\rangle=0
$$

So $(u, v)$ is a critical point of of $\mathcal{E}_{\gamma}$, that is $(u, v)$ is a weak solution of $(\mathcal{K S})_{\gamma}$.

We consider a new sequence $\left(\widetilde{u}_{n}, \widetilde{v}_{n}\right)=\left(u_{n}-u, v_{n}-v\right)$. Now we verify that

$$
\mathcal{E}_{\gamma}\left(\widetilde{u}_{n}, \widetilde{v}_{n}\right)=c-\mathcal{E}_{\gamma}(u, v) \text { as } n \rightarrow \infty
$$

and

$$
\mathcal{E}_{\gamma}^{\prime}\left(\widetilde{u}_{n}, \widetilde{v}_{n}\right) \rightarrow 0 \text { as } n \rightarrow \infty
$$

By the Brézis-Lieb lemma, we have that

$$
\left\|\left(\widetilde{u}_{n}, \widetilde{v}_{n}\right)\right\|_{\gamma}^{2}=\left\|\left(u_{n}, v_{n}\right)\right\|_{\gamma}^{2}-\|(u, v)\|_{\gamma}^{2}+o_{n}(1),
$$




$$
\begin{aligned}
& \left(\int_{\mathbb{R}^{3}}\left|\nabla \widetilde{u}_{n}\right|^{2} d x\right)^{2}=\left(\int_{\mathbb{R}^{3}}\left|\nabla u_{n}\right|^{2} d x\right)^{2}-\left(\int_{\mathbb{R}^{3}}|\nabla u|^{2} d x\right)^{2}+o_{n}(1), \\
& \left(\int_{\mathbb{R}^{3}}\left|\nabla \widetilde{v}_{n}\right|^{2} d x\right)^{2}=\left(\int_{\mathbb{R}^{3}}\left|\nabla v_{n}\right|^{2} d x\right)^{2}-\left(\int_{\mathbb{R}^{3}}|\nabla v|^{2} d x\right)^{2}+o_{n}(1) .
\end{aligned}
$$

To show (2.11) we observe

$$
\begin{aligned}
\mathcal{E}_{\gamma}\left(\widetilde{u}_{n}, \widetilde{v}_{n}\right)= & \frac{1}{2} \int_{\mathbb{R}^{3}}\left(a_{1}\left|\nabla \widetilde{u}_{n}\right|^{2}+\gamma V(x)\left|\widetilde{u}_{n}\right|^{2}\right) d x \\
& +\frac{1}{2} \int_{\mathbb{R}^{3}}\left(a_{2}\left|\nabla \widetilde{v}_{n}\right|^{2}+\gamma W(x)\left|\widetilde{v}_{n}\right|^{2}\right) d x \\
& +\frac{1}{4}\left(b_{1}\left(\int_{\mathbb{R}^{3}}\left|\nabla \widetilde{u}_{n}\right|^{2} d x\right)^{2}+b_{2}\left(\int_{\mathbb{R}^{3}}\left|\nabla \widetilde{v}_{n}\right|^{2} d x\right)^{2}\right) \\
& -\frac{2}{\alpha+\beta} \int_{\mathbb{R}^{3}}\left|\widetilde{u}_{n}\right|^{\alpha}\left|\widetilde{v}_{n}\right|^{\beta} d x \\
= & \mathcal{E}_{\gamma}\left(u_{n}, v_{n}\right)-\mathcal{E}_{\gamma}(u, v)+o_{n}(1) \\
& +\frac{2}{\alpha+\beta}\left(\int_{\mathbb{R}^{3}}\left|u_{n}\right|^{\alpha}\left|v_{n}\right|^{\beta} d x-\int_{\mathbb{R}^{3}}|u|^{\alpha}|v|^{\beta} d x-\left.\int_{\mathbb{R}^{3}}\left|\widetilde{u}_{n}\right|^{\alpha} \widetilde{v}_{n}\right|^{\beta} d x\right) .
\end{aligned}
$$

From Lemma 2.4, $\int_{\mathbb{R}^{3}}\left|u_{n}\right|^{\alpha}\left|v_{n}\right|^{\beta} d x-\int_{\mathbb{R}^{3}}|u|^{\alpha}|v|^{\beta} d x-\int_{\mathbb{R}^{3}}\left|\widetilde{u}_{n}\right|^{\alpha}\left|\widetilde{v}_{n}\right|^{\beta} d x \rightarrow 0$ as $n \rightarrow \infty$. Thus from (2.13) we obtain $(2.11)$.

In order to show $(2.12)$, let $(\varphi, \psi) \in E$. We note that

$$
\begin{aligned}
\left\langle\mathcal{E}_{\gamma}^{\prime}\left(\widetilde{u}_{n}, \widetilde{v}_{n}\right),(\varphi, \psi)\right\rangle= & \left\langle\mathcal{E}_{\gamma}^{\prime}\left(u_{n}, v_{n}\right),(\varphi, \psi)\right\rangle-\left\langle\mathcal{E}_{\gamma}^{\prime}(u, v),(\varphi, \psi)\right\rangle \\
& -\frac{2 \alpha}{\alpha+\beta} \int_{\mathbb{R}^{3}}\left|\widetilde{u}_{n}\right|^{\alpha-2}\left|\widetilde{v}_{n}\right|^{\beta} \widetilde{u}_{n} \varphi d x \\
& -\frac{2 \beta}{\alpha+\beta} \int_{\mathbb{R}^{3}}\left|\widetilde{u}_{n}\right|^{\alpha}\left|\widetilde{v}_{n}\right|^{\beta-2} \widetilde{v}_{n} \psi d x \\
& +\frac{2 \alpha}{\alpha+\beta} \int_{\mathbb{R}^{3}}\left|u_{n}\right|^{\alpha-2}\left|v_{n}\right|^{\beta} u_{n} \varphi d x \\
& +\frac{2 \beta}{\alpha+\beta} \int_{\mathbb{R}^{3}}\left|u_{n}\right|^{\alpha}\left|v_{n}\right|^{\beta-2} v_{n} \psi d x \\
& -\frac{2 \alpha}{\alpha+\beta} \int_{\mathbb{R}^{3}}|u|^{\alpha-2}|v|^{\beta} u \varphi d x \\
& -\frac{2 \beta}{\alpha+\beta} \int_{\mathbb{R}^{3}}|u|^{\alpha}|v|^{\beta-2} v \psi d x .
\end{aligned}
$$

Since $\mathcal{E}_{\gamma}^{\prime}\left(u_{n}, v_{n}\right) \rightarrow 0$ and $u_{n} \rightarrow u, v_{n} \rightarrow v$ in $L^{s}\left(\mathbb{R}^{3}\right)(2 \leq s<6)$, we have

$$
\begin{aligned}
& \lim _{n \rightarrow \infty} \sup _{\|\varphi\|_{\gamma, V} \leq 1} \int_{\mathbb{R}^{3}}\left(\left|\widetilde{u}_{n}\right|^{\alpha-2}\left|\widetilde{v}_{n}\right|^{\beta} \widetilde{u}_{n}-\left|u_{n}\right|^{\alpha-2}\left|v_{n}\right|^{\beta} u_{n}+|u|^{\alpha-2}|v|^{\beta} u\right) \varphi d x=0, \\
& \lim _{n \rightarrow \infty} \sup _{\|\psi\|_{\gamma, W} \leq 1} \int_{\mathbb{R}^{3}}\left(\left|\widetilde{u}_{n}\right|^{\alpha}\left|\widetilde{v}_{n}\right|^{\beta-2} \widetilde{v}_{n}-\left|u_{n}\right|^{\alpha}\left|v_{n}\right|^{\beta-2} v_{n}+|u|^{\alpha}|v|^{\beta-2} v\right) \psi d x=0 .
\end{aligned}
$$


Thus from (2.14) we obtain that

$$
\lim _{n \rightarrow \infty}\left\langle\mathcal{E}_{\gamma}^{\prime}\left(\widetilde{u}_{n}, \widetilde{v}_{n}\right),(\varphi, \psi)\right\rangle=0, \forall(\varphi, \psi) \in E,
$$

which implies (2.12) and this completes the proof of Lemma 2.5.

Then we have the following compactness result.

Lemma 2.6. Suppose that $\left(\mathcal{H}_{1}\right)$ and $\left(\mathcal{H}_{2}\right)$ hold. Then for any $C_{0}>0$, there exists $\Gamma_{0}>0$ such that $\mathcal{E}_{\gamma}$ satisfies the $(P S)_{c}$-condition for all $c \leq C_{0}$ and $\gamma \geq \Gamma_{0}$.

Proof. Let $c_{0}>0$ be given by Lemma 2.2(ii) and choose $\varepsilon>0$ such that $\varepsilon<\frac{c_{0}(\alpha+\beta)}{\alpha+\beta-2}$. Then for the given $C_{0}>0$, we choose $\Gamma_{\varepsilon}>0$ and $\rho_{\varepsilon}>0$ in Lemma 2.3. We claim that $\Gamma_{0}=\Gamma_{\varepsilon}$ is as required in Lemma 2.6. Let $\left\{\left(u_{n}, v_{n}\right)\right\} \subset E$ be a $(P S)_{c}$-sequence of $\mathcal{E}_{\gamma}(u, v)$ with $c \leq C_{0}$ and $\gamma \geq \Gamma_{0}$. By Lemma 2.5, we may suppose that $\left(u_{n}, v_{n}\right) \rightarrow(u, v)$ weakly in $E$ and then $\left\{\left(\widetilde{u}_{n}, \widetilde{v}_{n}\right)\right\}=\left\{\left(u_{n}-u, v_{n}-v\right)\right\}$ is a $(P S)_{d}$-sequence of $\mathcal{E}_{\gamma}$ with $d=c-\mathcal{E}_{\gamma}(u, v)$. We claim that $d=0$. Arguing by contradiction, assume that $d \neq 0$. Lemma 2.2 (ii) implies that $d \geq c_{0}>0$. Since $\left(\widetilde{u}_{n}, \widetilde{v}_{n}\right)$ is a $(P S)_{d}$-sequence of $\mathcal{E}_{\gamma}$, we have

$$
\mathcal{E}_{\gamma}\left(\widetilde{u}_{n}, \widetilde{v}_{n}\right)=d+o_{n}(1), \quad \mathcal{E}_{\gamma}^{\prime}\left(\widetilde{u}_{n}, \widetilde{v}_{n}\right)=o_{n}(1)
$$

Then we get

$$
\begin{aligned}
d+o_{n}(1)-\frac{1}{2} o_{n}\left(\left\|\left(u_{n}, v_{n}\right)\right\|_{\gamma}\right)= & \mathcal{E}_{\gamma}\left(\widetilde{u}_{n}, \widetilde{v}_{n}\right)-\frac{1}{2}\left\langle\mathcal{E}_{\gamma}^{\prime}\left(\widetilde{u}_{n}, \widetilde{v}_{n}\right),\left(\widetilde{u}_{n}, \widetilde{v}_{n}\right)\right\rangle \\
= & -\frac{1}{4}\left(b_{1} \Upsilon^{2}\left(\widetilde{u}_{n}\right)+b_{2} \Upsilon^{2}\left(\widetilde{v}_{n}\right)\right) \\
& +\left(1-\frac{2}{\alpha+\beta}\right) \int_{\mathbb{R}^{3}}\left|\widetilde{u}_{n}\right|^{\alpha}\left|\widetilde{v}_{n}\right|^{\beta} d x \\
\leq & \left(1-\frac{2}{\alpha+\beta}\right) \int_{\mathbb{R}^{3}}\left|\widetilde{u}_{n}\right|^{\alpha}\left|\widetilde{v}_{n}\right|^{\beta} d x .
\end{aligned}
$$

From (2.15) we deduce that

$$
\lim _{n \rightarrow \infty} \int_{\mathbb{R}^{3}}\left|\widetilde{u}_{n}\right|^{\alpha}\left|\widetilde{v}_{n}\right|^{\beta} d x \geq d\left(1-\frac{2}{\alpha+\beta}\right)^{-1} \geq \frac{\alpha+\beta}{\alpha+\beta-2} c_{0} .
$$

On the other hand, Lemma 2.3 implies

$$
\limsup _{n \rightarrow \infty} \int_{B_{\rho_{\varepsilon}}^{c}}\left|\widetilde{u}_{n}\right|^{\alpha}\left|\widetilde{v}_{n}\right|^{\beta} d x \leq \varepsilon<\frac{c_{0}(\alpha+\beta)}{\alpha+\beta-2} .
$$

This implies $\left(\widetilde{u}_{n}, \widetilde{v}_{n}\right) \rightarrow(\tilde{u}, \tilde{v})$ in $E$ with $(\tilde{u}, \tilde{v}) \neq(0,0)$, which is a contradiction. Therefore $d=0$ and it follows from (2.4) that

$$
\lim _{n \rightarrow \infty}\left\|\left(\widetilde{u}_{n}, \widetilde{v}_{n}\right)\right\|_{\gamma}^{2} \leq 2 d=0
$$

hence $\left(\widetilde{u}_{n}, \widetilde{v}_{n}\right) \rightarrow(0,0)$ in $E$, that is, $\left(u_{n}, v_{n}\right) \rightarrow(u, v)$ in $E$. This completes the proof of Lemma 2.6. 


\section{Proof of the main results}

In this section we give the proofs of our main results. First, we give the proof of Theorem 1.1.

Proof of Theorem 1.1. First, we can check that $\mathcal{E}_{\gamma}$ satisfies the mountain-pass geometry. Then using a version of the mountain-pass theorem without (PS) condition, there exists $\left\{\left(u_{n}, v_{n}\right)\right\} \subset E$ satisfying

$$
\mathcal{E}_{\gamma}\left(u_{n}, v_{n}\right) \rightarrow c_{\gamma} \text { and } \mathcal{E}_{\gamma}^{\prime}\left(u_{n}, v_{n}\right) \rightarrow 0 .
$$

Moreover, by Lemma 2.2(i) $\left\{\left(u_{n}, v_{n}\right)\right\}$ is bounded in $E$. Then, up to a subsequence, $\left(u_{n}, v_{n}\right) \rightarrow(u, v)$ weakly in $E$ and $\left(u_{n}, v_{n}\right) \rightarrow(u, v)$ a.e. in $x \in \mathbb{R}^{3}$. By Lemma 2.6, there exists $\gamma^{*}>0$, such that $\left(u_{n}, v_{n}\right) \rightarrow(u, v)$ in $E$ for $\gamma \geq \gamma^{*}$. Furthermore, by Lemma 2.5 we have that $\mathcal{E}_{\gamma}^{\prime}(u, v)=0$. By Lemma 2.1(i), we know that $(u, v) \neq(0,0)$, then $(u, v) \in \mathcal{N}_{\gamma}$, and using the Fatou's lemma we get

$$
\begin{aligned}
\mathcal{E}_{\gamma}(u, v) & =\mathcal{E}_{\gamma}(u, v)-\frac{1}{4}\left\langle\mathcal{E}_{\gamma}^{\prime}(u, v),(u, v)\right\rangle \\
& =\frac{1}{4}\|(u, v)\|_{\gamma}^{2}+\left(\frac{1}{2}-\frac{2}{\alpha+\beta}\right) \int_{\mathbb{R}^{3}}|u|^{\alpha}|v|^{\beta} d x \\
& \left.\leq \liminf _{n \rightarrow \infty}\left(\frac{1}{4}\left\|\left(u_{n}, v_{n}\right)\right\|_{\gamma}^{2}+\left(\frac{1}{2}-\frac{2}{\alpha+\beta}\right) \int_{\mathbb{R}^{3}}\left|u_{n}\right|^{\alpha}\left|v_{n}\right|^{\beta} d x\right)\right) \\
& =\liminf _{n \rightarrow \infty}\left(\mathcal{E}_{\gamma}\left(u_{n}, v_{n}\right)-\frac{1}{4}\left\langle\mathcal{E}_{\gamma}^{\prime}\left(u_{n}, v_{n}\right),\left(u_{n}, v_{n}\right)\right\rangle\right) \\
& =c_{\gamma} .
\end{aligned}
$$

Hence, $\mathcal{E}_{\gamma}(u, v) \leq c_{\gamma}$. On the other hand, from the definition of $c_{\gamma}$, we have $c_{\gamma} \leq \mathcal{E}_{\gamma}(u, v)$. So, $\mathcal{E}_{\gamma}(u, v)=c_{\gamma}$, that is $(u, v)$ is a ground state solution of problem $(\mathcal{K S})_{\gamma}$.

In order to investigate the concentration for the solutions obtained in Theorem 1.1, we consider the following Kirchhoff-type system:

$(\mathcal{K S})_{\infty} \quad \begin{cases}-\left(a_{1}+b_{1} \int_{\Omega}|\nabla u|^{2} d x\right) \Delta u=\frac{2 \alpha}{\alpha+\beta}|u|^{\alpha-2} u|v|^{\beta} & \text { in } \Omega, \\ -\left(a_{2}+b_{2} \int_{\Omega}|\nabla v|^{2} d x\right) \Delta v=\frac{2 \beta}{\alpha+\beta}|u|^{\alpha}|v|^{\beta-2} v & \text { in } \Omega, \\ u(x)=0, \quad v(x)=0 & \text { on } \partial \Omega,\end{cases}$

where $\Omega=\operatorname{int}\left(V^{-1}(0)\right)=\operatorname{int}\left(W^{-1}(0)\right)$. The energy functional associated with $(\mathcal{K S})_{\infty}$ is defined by

$$
\begin{aligned}
\mathcal{E}_{\infty}(u, v)= & \left.\frac{1}{2} \int_{\Omega}\left(a_{1}|\nabla u|^{2}+a_{2}|\nabla v|^{2}\right)\right) d x \\
& +\frac{1}{4}\left(b_{1}\left(\int_{\Omega}|\nabla u|^{2} d x\right)^{2}+b_{2}\left(\int_{\Omega}|\nabla v|^{2} d x\right)^{2}\right)-\frac{2}{\alpha+\beta} \int_{\Omega}|u|^{\alpha}|v|^{\beta} d x .
\end{aligned}
$$


Let

$$
\mathcal{M}:=\left\{(u, v) \in\left(H_{0}^{1}(\Omega) \times H_{0}^{1}(\Omega)\right) \backslash\{(0,0)\}:\left\langle\mathcal{E}_{\infty}^{\prime}(u, v),(u, v)\right\rangle=0\right\}
$$

be the manifold and $c^{\infty}=\inf _{(u, v) \in \mathcal{M}} \mathcal{E}_{\infty}(u, v)$.

Let us point out that the same results hold with $\mathcal{E}_{\infty}, c^{\infty}, \mathcal{M}$ in place of $\mathcal{E}_{\gamma}, c_{\gamma}, \mathcal{N}_{\gamma}$, respectively. We note that condition $\left(\mathcal{H}_{2}\right)$ implies that the Sobolev imbedding $H_{0}^{1}(\Omega) \times H_{0}^{1}(\Omega) \hookrightarrow L^{s_{1}}(\Omega) \times L^{s_{2}}(\Omega)$ is compact for $2 \leq s_{1}, s_{2}<6$, and hence the following Lemma 3.1 is standard.

Lemma 3.1. The infimum $c^{\infty}$ is achieved by a pair of functions $(u, v) \in \mathcal{M}$ which is a ground state solution of $(\mathcal{K S})_{\infty}$.

Lemma 3.2. $\lim _{\gamma \rightarrow+\infty} c_{\gamma}=c^{\infty}$, where $c_{\gamma}$ is defined in (1.4).

Proof. It is easy to see that $c_{\gamma} \leq c^{\infty}$ for all $\gamma \geq 0$. We assume $\lim _{n \rightarrow \infty} c_{\gamma_{n}}=$ $k<c^{\infty}$ for a sequence $\gamma_{n} \rightarrow \infty$ as $n \rightarrow \infty$. Lemma 2.2 implies $k>0$. We assume that $\left\{\left(u_{n}, v_{n}\right)\right\}$ such that $c_{\gamma_{n}}$ is achieved. By Lemma 2.2(i), $\left\{\left(u_{n}, v_{n}\right)\right\}$ is bounded in $E$, we may assume that

$$
\begin{cases}\left(u_{n}, v_{n}\right) \rightarrow(u, v), & \text { in } E, \\ \left(u_{n}, v_{n}\right) \rightarrow(u, v), & \text { in } L_{l o c}^{s_{1}}\left(\mathbb{R}^{3}\right) \times L_{l o c}^{s_{2}}\left(\mathbb{R}^{3}\right), 2 \leq s_{1}, s_{2}<6 .\end{cases}
$$

Now we claim that $\left.(u, v)\right|_{\Omega^{c}}=(0,0)$. In fact, if $\left.(u, v)\right|_{\Omega^{c}} \neq(0,0)$, there exists a compact subset $\mathcal{D} \subset \Omega^{c}$ with $\operatorname{dist}(\mathcal{D}, \partial \Omega)>0$ such that $\left.(u, v)\right|_{\mathcal{D}} \neq(0,0)$. Then by $(3.1)$

$$
\int_{\mathcal{D}}\left|u_{n}\right|^{2} d x \rightarrow \int_{\mathcal{D}}|u|^{2} d x>0, \quad \int_{\mathcal{D}}\left|v_{n}\right|^{2} d x \rightarrow \int_{\mathcal{D}}|v|^{2} d x>0 .
$$

Moreover, there exists $\varepsilon_{0}>0$ such that $V(x) \geq \varepsilon_{0}, W(x) \geq \varepsilon_{0}$ for any $x \in \mathcal{D}$. By the choice of $\left\{\left(u_{n}, v_{n}\right)\right\}$, we have

$$
\begin{aligned}
& \mathcal{E}_{\gamma_{n}}\left(u_{n}, v_{n}\right) \\
= & \frac{1}{2} \int_{\mathbb{R}^{3}}\left(a_{1}\left|\nabla u_{n}\right|^{2}+a_{2}\left|\nabla v_{n}\right|^{2}+\gamma_{n}\left(V(x) u_{n}^{2}+W(x) v_{n}^{2}\right)\right) d x \\
& +\frac{1}{4}\left(b_{1} \Upsilon^{2}\left(u_{n}\right)+b_{2} \Upsilon^{2}\left(v_{n}\right)\right)-\frac{2}{\alpha+\beta} \int_{\mathbb{R}^{3}}\left|u_{n}\right|^{\alpha}\left|v_{n}\right|^{\beta} d x \\
= & \left(\frac{1}{2}-\frac{1}{\alpha+\beta}\right) \int_{\mathbb{R}^{3}}\left(a_{1}\left|\nabla u_{n}\right|^{2}+a_{2}\left|\nabla v_{n}\right|^{2}+\gamma_{n}\left(V(x) u_{n}^{2}+W(x) v_{n}^{2}\right)\right) d x \\
& +\left(\frac{1}{4}-\frac{1}{\alpha+\beta}\right)\left(b_{1} \Upsilon^{2}\left(u_{n}\right)+b_{2} \Upsilon^{2}\left(v_{n}\right)\right) \\
\geq & \left(\frac{1}{2}-\frac{1}{\alpha+\beta}\right) \int_{\mathbb{R}^{3}}\left(\gamma_{n}\left(V(x) u_{n}^{2}+W(x) v_{n}^{2}\right)\right) d x \\
\geq & \left(\frac{1}{2}-\frac{1}{\alpha+\beta}\right) \int_{\mathcal{D}} \gamma_{n} \varepsilon_{0}\left(u_{n}^{2}+v_{n}^{2}\right) d x \rightarrow+\infty \text { as } n \rightarrow \infty .
\end{aligned}
$$

This contradiction shows that $\left.(u, v)\right|_{\Omega^{c}}=(0,0)$. 
Next we show that $u_{n} \rightarrow u, v_{n} \rightarrow v$ in $L^{q}\left(\mathbb{R}^{3}\right)$ for $2<q<6$. Otherwise, by concentration-compactness principle of P. L. Lions [11], there exist $\delta>0, \varrho>0$, $y_{n}, \tilde{y}_{n} \in \mathbb{R}^{3}$ with $\left|y_{n}\right| \rightarrow+\infty,\left|\tilde{y}_{n}\right| \rightarrow+\infty$ such that

$$
\begin{gathered}
\int_{B_{\varrho}\left(y_{n}\right)}\left|u_{n}-u\right|^{2} d x \geq \delta>0, \\
\int_{B_{\varrho}\left(\tilde{y}_{n}\right)}\left|v_{n}-v\right|^{2} d x \geq \delta>0 .
\end{gathered}
$$

On the other hand, by the choice of $\left\{\left(u_{n}, v_{n}\right)\right\}$ and the facts that $\mathcal{L}\left(B_{\varrho}\left(y_{n}\right) \cap\right.$ $\left.\left\{x \mid V(x) \leq M_{1}\right\}\right) \rightarrow 0, \mathcal{L}\left(B_{\varrho}\left(\tilde{y}_{n}\right) \cap\left\{x \mid W(x) \leq M_{2}\right\}\right) \rightarrow 0$ as $n \rightarrow \infty$, we obtain

$$
\begin{aligned}
& \mathcal{E}_{\gamma_{n}}\left(u_{n}, v_{n}\right) \\
\geq & \left(\frac{1}{2}-\frac{1}{\alpha+\beta}\right) \int_{B_{\varrho}\left(y_{n}\right) \cap\left\{x \mid V(x) \geq M_{1}\right\}}\left(a_{1}\left|\nabla u_{n}\right|^{2}+\gamma_{n} V(x) u_{n}^{2}\right) d x \\
& +\left(\frac{1}{2}-\frac{1}{\alpha+\beta}\right) \int_{B_{\varrho}\left(\tilde{y}_{n}\right) \cap\left\{x \mid W(x) \geq M_{2}\right\}}\left(a_{2}\left|\nabla v_{n}\right|^{2}+\gamma_{n} W(x) v_{n}^{2}\right) d x \\
\geq & \left(\frac{1}{2}-\frac{1}{\alpha+\beta}\right) \gamma_{n}\left(M_{1} \int_{B_{\varrho}\left(y_{n}\right)}\left|u_{n}-u\right|^{2} d x-\int_{B_{\varrho}\left(y_{n}\right) \cap\left\{x \mid V(x) \leq M_{1}\right\}}\left|u_{n}-u\right|^{2} d x\right) \\
& +\left(\frac{1}{2}-\frac{1}{\alpha+\beta}\right) \gamma_{n}\left(M_{2} \int_{B_{\varrho}\left(\tilde{y}_{n}\right)}\left|v_{n}-v\right|^{2} d x-\int_{B_{\varrho}\left(\tilde{y}_{n}\right) \cap\left\{x \mid W(x) \leq M_{2}\right\}}\left|v_{n}-v\right|^{2} d x\right) \\
= & \left(\frac{1}{2}-\frac{1}{\alpha+\beta}\right) \gamma_{n}\left(M_{1} \int_{B_{\varrho}\left(y_{n}\right)}\left|u_{n}-u\right|^{2} d x-o_{n}(1)\right) \\
& +\left(\frac{1}{2}-\frac{1}{\alpha+\beta}\right) \gamma_{n}\left(M_{2} \int_{B_{\varrho}\left(\tilde{y}_{n}\right)}\left|v_{n}-v\right|^{2} d x-o_{n}(1)\right) \rightarrow+\infty, \text { as } n \rightarrow \infty .
\end{aligned}
$$

This contradiction implies $u_{n} \rightarrow u, v_{n} \rightarrow v$ in $L^{q}\left(\mathbb{R}^{3}\right)$ for $2<q<6$.

Since $\left\{\left(u_{n}, v_{n}\right)\right\}$ is bounded in $E$, by the Fatou's lemma, we have that

$$
\begin{aligned}
& \liminf _{n \rightarrow \infty} \int_{\mathbb{R}^{3}}\left|\nabla u_{n}\right|^{2} d x \geq \int_{\mathbb{R}^{3}}|\nabla u|^{2} d x, \\
& \liminf _{n \rightarrow \infty} \int_{\mathbb{R}^{3}}\left|\nabla v_{n}\right|^{2} d x \geq \int_{\mathbb{R}^{3}}|\nabla v|^{2} d x .
\end{aligned}
$$

On the other hand, by the choice of $\left\{\left(u_{n}, v_{n}\right)\right\}$, we obtain

$$
\begin{aligned}
& \int_{\mathbb{R}^{3}}\left(a_{1}\left|\nabla u_{n}\right|^{2}+a_{2}\left|\nabla v_{n}\right|^{2}+\gamma_{n}\left(V(x) u_{n}^{2}+W(x) v_{n}^{2}\right)\right) d x \\
& +b_{1} \Upsilon^{2}\left(u_{n}\right)+b_{2} \Upsilon^{2}\left(v_{n}\right) \\
= & 2 \int_{\mathbb{R}^{3}}\left|u_{n}\right|^{\alpha}\left|v_{n}\right|^{\beta} d x .
\end{aligned}
$$

By (3.2) it follows that

$$
\int_{\mathbb{R}^{3}}\left(a_{1}|\nabla u|^{2}+a_{2}|\nabla v|^{2}\right) d x+b_{1} \Upsilon^{2}(u)+b_{2} \Upsilon^{2}(v)
$$




$$
\begin{aligned}
& \leq \lim _{n \rightarrow \infty}\left[\int_{\mathbb{R}^{3}}\left(a_{1}\left|\nabla u_{n}\right|^{2}+a_{2}\left|\nabla v_{n}\right|^{2}\right) d x+b_{1} \Upsilon^{2}\left(u_{n}\right)+b_{2} \Upsilon^{2}\left(v_{n}\right)\right] \\
& \leq 2 \lim _{n \rightarrow \infty} \int_{\mathbb{R}^{3}}\left|u_{n}\right|^{\alpha}\left|v_{n}\right|^{\beta} d x .
\end{aligned}
$$

Next, we first prove that

$$
\lim _{n \rightarrow \infty} \int_{\mathbb{R}^{3}}\left|u_{n}\right|^{\alpha}\left|v_{n}\right|^{\beta} d x=\int_{\mathbb{R}^{3}}|u|^{\alpha}|v|^{\beta} d x .
$$

Given $\varepsilon>0$, we can argue as in the proof of Lemma 2.3 to conclude that, for some $\rho>0$ large, there holds

$$
\limsup _{n \rightarrow \infty} \int_{B_{\rho}^{c}(0)}\left|u_{n}\right|^{\alpha}\left|v_{n}\right|^{\beta} d x \leq \varepsilon .
$$

By taking $\rho$ larger if necessary, we may assume that $\int_{B_{\rho}^{c}(0)}|u|^{\alpha}|v|^{\beta} d x \leq \varepsilon$. Moreover, the local convergence in (3.1) and the Lebesgue dominated convergence theorem imply that

Since

$$
\int_{B_{\rho}(0)}\left|u_{n}\right|^{\alpha}\left|v_{n}\right|^{\beta} d x \rightarrow \int_{B_{\rho}(0)}|u|^{\alpha}|v|^{\beta} d x \text { as } n \rightarrow \infty .
$$

$$
\begin{aligned}
& \left|\int_{\mathbb{R}^{3}}\left(\left|u_{n}\right|^{\alpha}\left|v_{n}\right|^{\beta}-|u|^{\alpha}|v|^{\beta}\right) d x\right| \\
\leq & \int_{B_{\rho}^{c}(0)}\left|u_{n}\right|^{\alpha}\left|v_{n}\right|^{\beta} d x+\int_{B_{\rho}^{c}(0)}|u|^{\alpha}|v|^{\beta} d x+\left|\int_{B_{\rho}(0)}\left(\left|u_{n}\right|^{\alpha}\left|v_{n}\right|^{\beta}-|u|^{\alpha}|v|^{\beta}\right) d x\right|,
\end{aligned}
$$

it follows from the above estimates and convergences that

$$
\limsup _{n \rightarrow \infty} \int_{\mathbb{R}^{3}}\left(\left|u_{n}\right|^{\alpha}\left|v_{n}\right|^{\beta}-|u|^{\alpha}|v|^{\beta}\right) d x \leq 2 \varepsilon,
$$

and therefore (3.4) holds. Then by (3.3) and (3.4) we have

$$
\int_{\mathbb{R}^{3}}\left(a_{1}|\nabla u|^{2}+a_{2}|\nabla v|^{2}\right) d x+b_{1} \Upsilon^{2}(u)+b_{2} \Upsilon^{2}(v) \leq 2 \int_{\mathbb{R}^{3}}|u|^{\alpha}|v|^{\beta} d x .
$$

As a consequence of $\left.(u, v)\right|_{\Omega^{c}}=(0,0)$ we obtain

$$
\begin{aligned}
& \int_{\Omega}\left(a_{1}|\nabla u|^{2}+a_{2}|\nabla v|^{2}\right) d x+b_{1}\left(\int_{\Omega}|\nabla u|^{2} d x\right)^{2}+b_{2}\left(\int_{\Omega}|\nabla v|^{2} d x\right)^{2} \\
\leq & 2 \int_{\Omega}|u|^{\alpha}|v|^{\beta} d x .
\end{aligned}
$$

Thus there exists $t_{0} \in(0,1]$ such that $t_{0}(u, v) \in \mathcal{M}$ and

$$
\mathcal{E}_{\infty}\left(t_{0}(u, v)\right) \leq \mathcal{E}_{\infty}(u, v),
$$

hence $c^{\infty} \leq \mathcal{E}_{\infty}\left(t_{0}(u, v)\right) \leq \mathcal{E}_{\infty}(u, v) \leq \lim _{n \rightarrow \infty} \mathcal{E}_{\gamma_{n}}\left(u_{n}, v_{n}\right)=k<c^{\infty}$, which is a contradiction. 
Now we give the proof of Theorem 1.2.

Proof of Theorem 1.2. For any sequence $\gamma_{n} \rightarrow+\infty$, let $\left(u_{n}, v_{n}\right):=\left(u_{\gamma_{n}}, v_{\gamma_{n}}\right)$ be the solutions of $(\mathcal{K S})_{\gamma_{n}}$ obtained in Theorem 1.1, that is $\gamma_{n} \rightarrow+\infty$ such that $\left(u_{n}, v_{n}\right) \in \mathcal{N}_{\gamma_{n}}, \mathcal{E}_{\gamma_{n}}\left(u_{n}, v_{n}\right)=c_{\gamma_{n}}$ and $\mathcal{E}_{\gamma_{n}}^{\prime}\left(u_{n}, v_{n}\right)=0$. By Lemma 2.2(i) we know that $\left\{\left(u_{n}, v_{n}\right)\right\}$ must be bounded in $H^{1}\left(\mathbb{R}^{3}\right) \times H^{1}\left(\mathbb{R}^{3}\right)$, we may assume that $\left(u_{n}, v_{n}\right) \rightarrow(u, v)$ in $H^{1}\left(\mathbb{R}^{3}\right) \times H^{1}\left(\mathbb{R}^{3}\right)$ and $\left(u_{n}, v_{n}\right) \rightarrow(u, v)$ in $L_{\text {loc }}^{s_{1}}\left(\mathbb{R}^{3}\right) \times L_{\text {loc }}^{s_{2}}\left(\mathbb{R}^{3}\right)$ for $2 \leq s_{1}, s_{2}<6$. We shall show that $(u, v) \in H_{0}^{1}(\Omega) \times$ $H_{0}^{1}(\Omega)$ is a ground state solution of $(\mathcal{K S})_{\infty}$, that is $\mathcal{E}_{\infty}(u, v)=c^{\infty},\left(u_{n}, v_{n}\right) \rightarrow$ $(u, v)$ in $H^{1}\left(\mathbb{R}^{3}\right) \times H^{1}\left(\mathbb{R}^{3}\right)$. As in the proof of Lemma 3.2 we can prove that $\left.(u, v)\right|_{\Omega^{c}}=(0,0)$ whereas $(u, v) \in H_{0}^{1}(\Omega) \times H_{0}^{1}(\Omega)$ and $\left(u_{n}, v_{n}\right) \rightarrow(u, v)$ in $L^{s_{1}}\left(\mathbb{R}^{3}\right) \times L^{s_{2}}\left(\mathbb{R}^{3}\right)$ for $2 \leq s_{1}, s_{2}<6$. Then it suffices to show that

$$
\begin{gathered}
\lim _{n \rightarrow \infty} \int_{\mathbb{R}^{3}}\left|\nabla u_{n}\right|^{2} d x=\int_{\mathbb{R}^{3}}|\nabla u|^{2} d x, \quad \lim _{n \rightarrow \infty} \int_{\mathbb{R}^{3}}\left|\nabla v_{n}\right|^{2} d x=\int_{\mathbb{R}^{3}}|\nabla v|^{2} d x, \\
\lim _{n \rightarrow \infty} \gamma_{n} \int_{\mathbb{R}^{3}} V(x) u_{n}^{2} d x=0, \quad \lim _{n \rightarrow \infty} \gamma_{n} \int_{\mathbb{R}^{3}} W(x) v_{n}^{2} d x=0 .
\end{gathered}
$$

In fact, if one of the above limits does not hold, by the Fatou's lemma, we have

$$
\int_{\mathbb{R}^{3}}\left(a_{1}|\nabla u|^{2}+a_{2}|\nabla v|^{2}\right) d x+b_{1} \Upsilon^{2}(u)+b_{2} \Upsilon^{2}(v)<2 \int_{\mathbb{R}^{3}}|u|^{\alpha}|v|^{\beta} d x,
$$

similar to the proof of Lemma 3.2, there exists $t_{0} \in(0,1)$ such that $t_{0}(u, v) \in \mathcal{M}$ and

$$
c^{\infty} \leq \mathcal{E}_{\infty}\left(t_{0}(u, v)\right)<\mathcal{E}_{\infty}(u, v) \leq \lim _{n \rightarrow \infty} \mathcal{E}_{\gamma_{n}}\left(u_{n}, v_{n}\right) \leq c^{\infty},
$$

which is a contradiction. This completes the proof of Theorem 1.2.

Acknowledgements. The author would like to thank the referees for valuable comments and helpful suggestions. This work is supported by the science and technology research project of Hubei Provincial Department of Education (D20142702).

\section{References}

[1] C. O. Alves, F. J. S. A. Correa, and T. F. Ma, Positive solutions for a quasilinear elliptic equation of Kirchhoff type, Comput. Math. Appl. 49 (2005), no. 1, 85-93.

[2] T. Bartsch and Z. Q. Wang, Multiple positive solutions for a nonlinear Schrödinger equation, Z. Angew. Math. Phys. 51 (2000), no. 3, 366-384.

[3] F. Cammaroto and L. Vilasi, Multiple solutions for a Kirchhoff-type problem involving the $p(x)$-Laplacian operator, Nonlinear Anal. 74 (2011), no. 5, 1841-1852.

[4] - On a Schrödinger-Kirchhoff-type equation involving the $p(x)$-Laplacian, Nonlinear Anal. 81 (2013), 42-53.

[5] B. T. Cheng and X. Wu, Existence results of positive solutions of Kirchhoff type problems, Nonlinear Anal. 71 (2009), no. 10, 4883-4892.

[6] M. F. Furtado, E. A. B. Silva, and M. S. Xavier, Multiplicity and concentration of solutions for elliptic systems with vanishing potentials, J. Differential Equations 249 (2010), no. 10, 2377-2396.

[7] X. M. He and W. M. Zou, Infinitely many positive solutions for Kirchhoff-type problems, Nonlinear Anal. 70 (2009), no. 3, 1407-1414. 
[8] — Existence and concentration behavior of positive solutions for a Kirchhoff equation in $\mathbb{R}^{3}$, J. Differential Equations 252 (2012), no. 2, 1813-1834.

[9] G. Kirchhoff, Mechanik, Teubner, Leipzig, 1883.

[10] J. L. Lions, On some questions in boundary value problems of mathematical physics, Contemporary developments in continuum mechanics and partial differential equations (Proc. Internat. Sympos., Inst. Mat., Univ. Fed. Rio de Janeiro, Rio de Janeiro, 1977), pp. 284-346, North-Holland Math. Stud., 30, North-Holland, Amsterdam-New York, 1978.

[11] P. L. Lions, The concentration-compactness principle in the calculus of variations. The locally compact case. Part I, Ann. Inst. H. Poincaré Anal. Non Linéaire 1 (1984), no. 2, 109-145.

[12] W. Liu and X. M. He, Multiplicity of high energy solutions for superlinear Kirchhoff equations, J. Appl. Math. Comput. 39 (2012), no. 1-2, 473-487.

[13] T. F. Ma and J. E. Munoz Rivera, Positive solutions for a nonlinear nonlocal elliptic transmission problem, Appl. Math. Lett. 16 (2003), no. 2, 243-248.

[14] A. M. Mao and Z. T. Zhang, Sign-changing and multiple solutions of Kirchhoff type problems without the P.S. condition, Nonlinear Anal. 70 (2009), no. 3, 1275-1287.

[15] K. Perera and Z. T. Zhang, Nontrivial solutions of Kirchhoff-type problems via the Yang index, J. Differential Equations 221 (2006), no. 1, 246-255.

[16] J. T. Sun and T. F. Wu, Ground state solutions for an indefinite Kirchhoff type problem with steep potential well, J. Differential Equations 256 (2014), no. 4, 1771-1792.

[17] J. Wang, L. Tian, J. Xu, and F. Zhang, Multiplicity and concentration of positive solutions for a Kirchhoff type problem with critical growth, J. Differential Equations 253 (2012), no. 7, 2314-2351.

[18] X. Wu, Existence of nontrivial solutions and high energy solutions for SchrödingerKirchhoff-type equations in $R^{N}$, Nonlinear Anal. Real World Appl. 12 (2011), no. 2, 1278-1287.

[19] High energy solutions of systems of Kirchhoff-type equations in $R^{N}$, J. Math. Phys. 53 (2012), no. 6, 063508, 18 pp.

[20] F. Zhou, K. Wu, and X. Wu, High energy solutions of systems of Kirchhoff-type equations on $R^{N}$, Comput. Math. Appl. 66 (2013), no. 7, 1299-1305.

School of Mathematics and Statistics

Hubei EngINEERING UNIVERSity

XiaOgan 432000, P. R. China

AND

School of Mathematics And Statistics

Central China Normal University

WuHAN 430079, P. R. CHinA

E-mail address: dengfeng1214@163.com 